\title{
Imatinib mesylate-induced generalized hypopigmentation in patients with chronic myeloid leukemia
}

Imatinib mesylate (STI-571) is a recently developed oral anticancer agent rationally designed to selectively inhibit certain protein kinases implicated in the pathogenesis of chronic myeloid leukemia (CML) and malignant gastrointestinal stromal tumors. ${ }^{[1]}$ Imatinib mesylate inhibits the bcr-abl tyrosine kinase, thus blocking proliferation and growth of tumor cells expressing bcr-abl. It is generally well tolerated and most side effects are mild to moderate in severity. The common side effects are edema, nausea, vomiting, diarrhea, muscle cramps, and cutaneous reactions. Serious side effects such as hepatotoxicity and myelosuppression also occur, warranting regular monitoring and interruption of treatment. ${ }^{[2]}$ The common dermatological side effects are periorbital edema and dermatitis. Facial edema, pruritis, erythema, dry skin, alopecia, night sweats, and photosensitivity reactions are infrequently reported. ${ }^{[3]}$ We are highlighting an interesting dermatological effect, generalized hypopigmentation, as a result of imatinib therapy, noticeable especially in Indian population because of the dark complexion.

A retrospective analysis of 15 patients of CML on imatinib mesylate between Sept 2003 and Sept 2004 was carried out. Imatinib mesylate was started as first-line therapy in five patients. In the remaining 10 patients, the drug was initiated when therapy with hydroxyurea and interferon did not show evidence of hematologic and cytogenetic response. Imatinib mesylate was initiated during the chronic phase of CML in eleven, accelerated phase in one, and blast crisis in three patients. The dose of imatinib was $400 \mathrm{mg} /$ day in the chronic phase and $600 \mathrm{mg} /$ day in the accelerated and blast crisis phases.

After initiation of the drug, patients were followed weekly for 1 month and thereafter every 2 weeks for an average of 15 months (range: 8-23 months). No patient was lost to follow up. During each visit, patients were assessed for constitutional symptoms, weight gain, periorbital edema, jaundice, vomiting, and diarrhea. Hemogram was done during each visit and liver function tests were done monthly. Bone marrow aspiration was done after 6 months of initiation of drug to assess the cytogenetic response. Complete hematological response was defined as disappearance of signs and symptoms of disease, no splenomegaly, and normal blood counts (TLC < 11000/ $\mathrm{mm}^{3}$, platelet count $<450000 / \mathrm{mm}^{3}$, and no immature white blood cells in smear). Cytogenetic responses were classified into major and minor responses. The major response was further classified as complete $(0 \%$ Philadelphia $[\mathrm{Ph}]$

\section{Table 1}

Summary of patients' characteristics

\begin{tabular}{|c|c|c|c|c|c|c|}
\hline Age & Sex & CML stage & Oral dose (mg/day) & First line drug ${ }^{a}$ & CML response & $\begin{array}{l}\text { eralized } \\
\text { ymentation }\end{array}$ \\
\hline 30 & $\mathrm{M}$ & Chronic & 400 & Imatinib & $\mathrm{HR}$ & Yes \\
\hline 35 & $\mathrm{~F}$ & Chronic & 400 & Hydroxyurea $^{b}$ & $\mathrm{HR}$ & Yes \\
\hline 28 & $\mathrm{M}$ & Chronic & 400 & Hydroxyurea $^{b}$ & $\mathrm{HR}+\mathrm{CCR}$ & Yes \\
\hline 40 & $\mathrm{M}$ & Chronic & 400 & Interferon ${ }^{b}$ & $\mathrm{HR}+\mathrm{CCR}$ & Yes \\
\hline 62 & $\mathrm{~F}$ & Chronic & 400 & Interferon ${ }^{b}$ & $\mathrm{HR}$ & Yes \\
\hline 38 & $\mathrm{~F}$ & Chronic & 400 & Hydroxyurea $^{b}$ & $\mathrm{HR}+\mathrm{PCR}$ & Yes \\
\hline 42 & $\mathrm{M}$ & Blast crisis & 600 & Imatinib & $\mathrm{HR}$ & No \\
\hline 45 & $\mathrm{M}$ & Blast Crisis & 600 & Imatinib & $\mathrm{HR}+\mathrm{CCR}$ & Yes \\
\hline 58 & $\mathrm{M}$ & Chronic & 400 & Interferon ${ }^{b}$ & $\mathrm{HR}$ & Yes \\
\hline 44 & $\mathrm{~F}$ & Chronic & 400 & Interferon & $\mathrm{HR}+\mathrm{PCR}$ & Yes \\
\hline 31 & $\mathrm{M}$ & Chronic & 400 & Interferon & $\mathrm{HR}$ & No \\
\hline 52 & $\mathrm{~F}$ & Chronic & 400 & Hydroxyurea $^{b}$ & $\mathrm{HR}+\mathrm{MCR}$ & Yes \\
\hline 30 & $\mathrm{M}$ & Blast crisis & 600 & Imatinib & Withdrawal of drug & No \\
\hline 36 & $\mathrm{~F}$ & Accelerated & 600 & Imatinib & -do- & Yes \\
\hline 23 & $\mathrm{M}$ & Chronic & 400 & Interferon ${ }^{b}$ & -do- & Yes \\
\hline
\end{tabular}

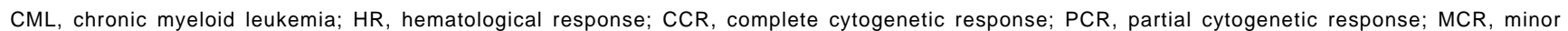

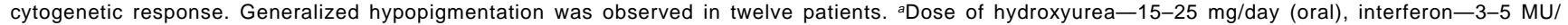
$\mathrm{m}^{2} /$ day subcutaneously. ${ }^{b}$ Imatinib was given as a second-line therapy. 
chromosome) and partial (0-34\% $\mathrm{Ph}$ chromosome). Minor response was defined as presence of $\mathrm{Ph}$ chromosome in 35$90 \%$ of metaphases.

Adverse effects owing to imatinib included mild constitutional symptoms (fever and arthralgia) in all and weight gain (range: $3-5 \mathrm{~kg}$ ) in six patients. No patient developed hepatotoxicity. Three patients had definite evidence of severe myelosuppression (TLC $<1000 / \mathrm{mm}^{3}$, normal: 4000 $11000 / \mathrm{mm}^{3}$, and a platelet count of less than $20000 / \mathrm{mm}^{3}$, normal: $150000-450000 / \mathrm{mm}^{3}$ ), necessitating withdrawal of the drug. These patients were followed weekly till recovery of counts (range: $3-6$ weeks), when the drug was restarted with a dose of $300 \mathrm{mg} /$ day. All patients showed complete hematological response, but complete cytogenetic response was seen in three, partial response in two, and a minor response in one patient (Table 1). Interestingly, we noticed the generalized lightening of skin color in 12 patients within 3-4 weeks of initiation of the drug. None of the patients reported the awareness of fairness of their complexion on their own but agreed with our observation when brought to their notice. Most of the patients and their immediate relatives felt that patients were never so fair even before detection of CML. Skin biopsy of all patients showed decreased pigmentation of epidermis, evidence of pigment incontinence, and dermal edema.

This interesting finding of generalized hypopigmentation appears to be reversible because withdrawal of the drug in patients showing severe myelosuppression led to the darkening of skin color and reinitiation of therapy reversed the skin darkening.

Imatinib mesylate has become the most effective initial treatment in bcr-abl-positive CML who do not have a suitable bone marrow donor or who are not candidates for transplantation. ${ }^{[2]}$ Recent studies demonstrated that imatinibtreated $(400 \mathrm{mg} /$ day) patients had significantly higher rates $(P<0.001)$ of complete hematological and cytogenetic response $^{[2]}$ compared with those receiving the combination of interferon $\left(5 \mathrm{MU} / \mathrm{m}^{2} /\right.$ day) plus cytarabine (Ara-C, $20 \mathrm{mg}$ / $\mathrm{m}^{2} /$ day).

Hypopigmentation owing to imatinib in CML patients has been reported only recently, especially in brown- or darkskinned races. ${ }^{[2,3]}$ Imatinib is an inhibitor of bcr-abl tyrosine kinase, platelet-derived growth factor receptor, and $C$-kit receptor tyrosine kinase. The last two kinases have critical roles in normal pigmentation. Mutation in these genes, reducing or abrogating the respective enzyme activities, are associated with loss of pigmentation owing to interruption of the development and differentiation of melanocytes. Imatinib produces a similar effect by inhibition of the crucial kinases. Rapid clearance of hydroxyurea from the hyperpigmented skin in four patients who had received the drug as the firstline therapy superimposed by decreased production of melanin as a result of initiation of imatinib mesylate led to a more dramatic effect in this group of patients. Hydroxyurea is a drug known to cause hyperpigmentation of skin and soft tissues. Review of literature revealed few case reports of imatinib-induced patchy as well as generalized hypopigmentation. ${ }^{[1,3]}$ Adverse effects arising from skin hypopigmentation include photosensitivity, which is resolved using a sun-screen and avoidance of sun exposure. ${ }^{[4]}$

In conclusion, we highlight an interesting dermatological effect-the generalized hypopigmentation, owing to imatinib mesylate that was noticed in $80 \%$ of our patients of CML. Although a cosmetic problem, it does not pose a substantial medical hazard and does not appear to predict the leukemic cell response or clinical outcome.

\section{Sunita, D. K. Gupta, S. Saluja*, S. Bhasin, M. Sharma*}

'Departments of Medicine and *Hematology, VMMC and Safdarjung Hospital, New Delhi 26166443, India. E-mail: sunitaamite@yahoo.co.in

\section{References}

1. Hasan S, Dinh K, Lombardo F, Dawkins F, Kark J. Hypopigmentation in an African patient treated with imatinib mesylate: A case report. J Natl Med Assoc 2003;95:722-4.

2. Guilhot $F$. Indications for imatinib mesylate therapy and clinical management. Oncologist 2004;9:271-81.

3. Sharma A, Vora A, Bhutani M. Generalized hypopigmentation due to imatinib: a fairness boon? Indian J Dermatol Venereol Leprol 2005;71:45-6.

4. Tsao AS, Kantarjian H, Cortes J, O'Brien S, Talpaz M. Imatinib mesylate causes hypopigmentation in the skin. Cancer 2003;98:2483-7.

\section{Join "IndPharm" \\ IJP uses "IndPharm" to broadcast announcements. Want to join? Please E-mail: adithan@vsnl.com}

